\title{
Vitamin D and assisted reproductive treatment outcome: a prospective cohort study
}

\author{
Justin Chu ${ }^{1,2}$, loannis Gallos ${ }^{1,2,4^{*}}$ (D), Aurelio Tobias ${ }^{1,3}$, Lynne Robinson², Jackson Kirkman-Brown ${ }^{1,2}$, \\ Rima Dhillon-Smith ${ }^{1,2}$, Hoda Harb ${ }^{1,2}$, Abey Eapen ${ }^{1,2}$, Madhurima Rajkhowa ${ }^{2}$ and Arri Coomarasamy ${ }^{1,2}$
}

\begin{abstract}
Background: Vitamin D deficiency has been associated with an increased risk of abnormal pregnancy implantation leading to obstetric complications such as pre-eclampsia and fetal growth restriction. However, the effect of vitamin D on reproductive treatment outcomes in couples undergoing assisted reproductive treatment is poorly understood. This study investigates the association between vitamin $\mathrm{D}$ and reproductive treatment outcomes in women undergoing assisted reproductive treatments?
\end{abstract}

Methods: A prospective cohort study conducted at a large tertiary teaching hospital, United Kingdom. Five hundred women undergoing assisted reproductive treatment were recruited between September 2013 and September 2015. All participants had their serum vitamin D measured and their reproductive treatment outcomes collated. Women were categorised in to three groups: vitamin D replete $(>75 \mathrm{nmol} / \mathrm{L})$, insufficient $(50-75 \mathrm{nmol} / \mathrm{L})$ and deficient $(<50$ $\mathrm{nmol} / \mathrm{L}$ ) according to Endocrine Society guidance. The primary outcome was live birth. Secondary outcomes included biochemical pregnancy, clinical pregnancy and pregnancy loss rates.

Results: Vitamin D deficiency was found in 53.2\% (266/500) of participants and vitamin D insufficiency was found in $30.8 \%(154 / 500)$ of participants. Only 16\% (80/500) of women were vitamin D replete. The live birth rates for vitamin D deficient, insufficient and replete women were 23.2\% (57/246), 27.0\% (38/141) and 37.7\% (29/77) respectively ( $p=0.04)$. The respective live birth rates for vitamin $D$ deficient, insufficient and replete women were $24.3,27.1,34.4 \%$ after adjustment for key prognostic factors $(p=0.25)$.

Conclusions: Vitamin D deficiency and insufficiency are common in women undergoing assisted reproductive treatments. The crude live birth rate achieved in women undergoing assisted reproductive treatments are associated with serum vitamin D, although statistical significance is lost when adjusting for important prognostic variables. Vitamin D deficiency could be an important condition to treat in women considering fertility treatment. A research trial to investigate the benefits of vitamin D deficiency treatment would test this hypothesis.

Trial registration: Clinicaltrials.gov - NCT02187146.

Keywords: Vitamin D, Implantation, Assisted reproductive treatment

\footnotetext{
* Correspondence: i.d.gallos@bham.ac.uk

${ }^{1}$ Tommy's National Centre for Miscarriage Research, Institute of Metabolism

and Systems Research (IMSR), University of Birmingham, Birmingham B15

$2 \pi$, UK

${ }^{2}$ Birmingham Women's and Children's National Foundation Trust,

Mindelsohn Way, Birmingham B15 2TG, UK

Full list of author information is available at the end of the article
}

(c) The Author(s). 2019 Open Access This article is distributed under the terms of the Creative Commons Attribution 4.0 International License (http://creativecommons.org/licenses/by/4.0/), which permits unrestricted use, distribution, and reproduction in any medium, provided you give appropriate credit to the original author(s) and the source, provide a link to the Creative Commons license, and indicate if changes were made. The Creative Commons Public Domain Dedication waiver (http://creativecommons.org/publicdomain/zero/1.0/) applies to the data made available in this article, unless otherwise stated. 


\section{Plain English summary}

This study investigates the association between vitamin $\mathrm{D}$ and reproductive treatment outcomes in women undergoing assisted reproductive treatments. The study comprises 500 women undergoing in vitro fertilization treatment at a large teaching hospital in the United Kingdom. It is thought that vitamin D is important for the implantation of an embryo to the lining of the womb and deficiency of vitamin D may reduce the chances of pregnancy from in vitro fertilization treatment. Vitamin $\mathrm{D}$ is categorized in to three groups; normal vitamin $\mathrm{D}$, insufficient vitamin $\mathrm{D}$ and deficient vitamin $\mathrm{D}$. Blood was taken prior to the participants' treatment and their pregnancy outcomes from their treatments were collated to investigate whether the vitamin status of the women was associated with the rate of implantation, clinical pregnancy, live birth and miscarriage. We found that vitamin $\mathrm{D}$ deficiency was common amongst our participants. Furthermore, an association was found between vitamin $\mathrm{D}$ status and chances of achieving pregnancy and also live birth. However, when important factors were controlled for, the results lost their statistical significance, although the highest rates of pregnancy were still found in the normal vitamin D group and the lowest rates of pregnancy were found in the deficient group. Vitamin D deficiency could be a condition that could be easily treated in women who are planning to undergo in vitro fertilization treatment for relatively low cost. A trial of vitamin D deficiency treatment should be conducted in women undergoing in vitro fertilization treatment to test this idea.

\section{Background}

Infertility affects one in seven couples in the United Kingdom (UK). In 2014, 52,288 women underwent assisted reproductive treatment (ART) cycles [1]. The overall success rate of these treatments was $36.3 \%$ [1]. In vitro fertilisation (IVF) success rates have gradually increased over time due to research in embryology, which has enhanced our abilities to select and transfer the embryo with the highest pregnancy potential [2]. More recently, the rate of improvement in success rates has slowed due to our inability to improve the likelihood of embryo implantation [3].

There has been recent interest in the role of vitamin D in reproductive physiology as vitamin D deficiency has been found to be common in women of resproductive age [4-6]. It is postulated that vitamin D is important in pregnancy implantation as vitamin $\mathrm{D}$ enzymes and receptors have been found in the endometrium [7]. Additionally, vitamin D deficiency has been found to reduce fertility capacity in animal studies [8-11]. In humans, the importance of vitamin D in placental function is the most studied aspect of vitamin D in reproduction [12].
Specifically, vitamin D deficiency has been linked to poor placentation and fetal growth restriction [12]. More recently, it has been proposed that vitamin D deficiency leads to improper embryo implantation causing poor placentation [13-15].

The majority of the body's vitamin D is in the form of vitamin D3 (cholecalciferol), which is photo-chemically synthesised in the skin [16]. Vitamin D levels are usually measured by assay of serum 25-hydroxy vitamin $\mathrm{D}_{3}$ concentration. People are at risk of the detrimental effects of vitamin $\mathrm{D}$ deficiency at serum 25 -hydroxy vitamin $\mathrm{D}_{3}$ concentrations of less than $50 \mathrm{nmol} / \mathrm{L}$ (less than $20 \mathrm{ng} /$ $\mathrm{mL}$ ). A level of 50 to $75 \mathrm{nmol} / \mathrm{L}$ ( 21 to $29 \mathrm{ng} / \mathrm{mL}$ ) is considered insufficient and greater than $75 \mathrm{nmol} / \mathrm{L}$ (greater than $30 \mathrm{ng} / \mathrm{ml}$ ) is considered vitamin D replete [17]. Serum concentrations greater than $374 \mathrm{nmol} / \mathrm{L}$ (greater than $150 \mathrm{ng} / \mathrm{mL}$ ) are associated with toxicity [6, 18-20].

The postulated role of vitamin $\mathrm{D}$ in implantation has led research groups to investigate the importance of vitamin $\mathrm{D}$ in patients undergoing IVF with conflicting results. None of these studies have been conducted in the UK. In this study, we prospectively examined vitamin D levels of a diverse population of women undergoing ART at an assisted conception unit in Birmingham, UK to identify whether there is an association between serum blood levels of 25-hydroxy vitamin $\mathrm{D}_{3}$ and fertility treatment outcomes.

\section{Methods}

The study was funded by the Birmingham Women's and Children's NHS Foundation Trust Research and Development Department and was approved by the National Research Ethics Service (NRES) Committee West Midlands - Black Country (REC 13/WM/0258). A total of 504 patients who underwent ART at the Birmingham Women's Fertility Centre from September 2013 to September 2015 were recruited.

All patients who were referred for IVF, intracytoplasmic sperm injection (ICSI) and frozen embryo transfer (FET) treatment met the inclusion criteria and were approached to participate. The only exclusion criterion was that patients were not recruited if they had already participated in the study in a previous IVF treatment cycle. Prospective participants were approached at their treatment consent signing appointment. Vitamin D assay for total 25-hydroxy vitamin D, 25-hydroxy vitamin $\mathrm{D}_{2}$ and 25-hydroxy vitamin $\mathrm{D}_{3}$ were measured. Assays used a liquid-liquid extraction method using highly sensitive liquid chromatography mass spectrometry (Waters Premier XE MS detector with ACQUITY Ultra Performance LC). Patients were allocated into three groups according to their total 25-hydroxy vitamin D levels; vitamin D deficient group (25-hydroxy vitamin D less than $50 \mathrm{nmol} / \mathrm{L}$ ), vitamin D insufficient group (25- 
hydroxy vitamin D 50-75 nmol/L) and vitamin D replete group (25-hydroxy greater than $75 \mathrm{nmol} / \mathrm{L}$ ) according to the Endocrine Society definitions [17].

\section{IVF, ICSI and FET treatments}

All participants undergoing fresh cycle IVF or ICSI underwent pituitary suppression using either a short antagonist down regulation protocol or a long down regulation protocol using Cetrotide (Merck Serono, France) or Buserelin (Sanofi-Aventis, France) respectively. After complete pituitary suppression, participants underwent ovarian stimulation with recombinant FSH (Gonal-F [Merck Serono, France] or Menopur [Ferring, France]). Starting doses depended on the patient's age and early follicular phase pituitary follicular stimulating hormone (FSH) level. Doses of exogenous FSH were then modified according to the patient's ovarian response tracked by transvaginal ultrasonography. The endometrial thickness was also measured at each scan. When at least three follicles reached a diameter of at least $18 \mathrm{~mm}$, $6500 \mathrm{IU}$ of Human Chorionic Gonadotrophin was used to trigger ovulation. In the event of ovarian hyperstimulation or inadequate ovarian stimulation, the treatment was abandoned if clinically required. In women with a suitable ovarian response to exogenous FSH, transvaginal oocyte retrieval was performed $36 \mathrm{~h}$ after trigger injection. Morphological grade of all embryos was assessed on days two, three and five post oocyte retrieval. Embryos were graded according to the degree of fragmentation and the regularity of blastomeres. Day five blastocyst grading was according to the development stage, inner cell mass quality and trophectoderm quality. Fresh embryo transfer was conducted in line with the Birmingham Women's Fertility Centre single embryo replacement policy. Endometrial support was achieved using Gestone injections (Nordic Pharma, France) or Cyclogest pessaries (Actavis, UK) commenced at the time of embryo transfer.

All participants having frozen embryo transfer (FET) treatment underwent pituitary suppression using Buserelin (Sanofi-Aventis, France) followed by oestrogen (Progynova [Bayer, UK] and progesterone administration in the form of Gestone injections (Nordic Pharma, France) or Cyclogest pessaries (Actavis, UK) to prepare the endometrium prior to embryo replacement.

\section{Outcome measures}

The primary outcome was live birth. Secondary reproductive treatment outcomes included biochemical pregnancy rates (positive pregnancy test rates 16 days after oocyte retrieval in fresh IVF or ICSI and two weeks after embryo replacement in FET), clinical pregnancy (presence of a fetal heartbeat on ultrasound scan performed five weeks after embryo replacement) and miscarriage rates. Secondary fertility cycle outcomes included FSH dose requirements, endometrial thickness at last monitoring pelvic ultrasound, number of oocytes retrieved, and quality of embryo/s replaced.

\section{Statistical analysis}

We aimed to recruit 490 patients undergoing IVF treatment (including IVF, ICSI treatment and FET). We hypothesised a pregnancy rate of $35 \%$ for women with replete serum vitamin D and $25 \%$ for patients with insufficient or deficient serum vitamin D levels. This gives an absolute risk difference of $10 \%$. A $10 \%$ attrition rate was anticipated. Aiming for $90 \%$ power with a $5 \%$ type two error rate, the sample size required was calculated as 490 .

The baseline characteristics, ART cycle characteristics, and reproductive treatment outcomes for the vitamin D deficient, insufficient and replete groups were analysed using Mann-Whitney U-tests for non-parametric data and Pearson $\times 2$ tests for categorical data.

Multivariable logistic regression was used to evaluate predictors of live birth, biochemical pregnancy and clinical pregnancy rates. Vitamin D status (deficient, sufficient, replete) was included in the model as an ordinal variable. Variables evaluated as potential confounders included age, body mass index, ethnicity, smoking status, cause of infertility, duration of infertility, baseline pituitary follicular stimulating hormone assay, treatment protocol, and type of treatment. Only those covariates that confounded the relationship between vitamin $\mathrm{D}$ and reproductive treatment outcome were included in the adjusted model. Model fit was evaluated using the Hosmer and Lemeshow test. Covariate-adjusted pregnancy and birth rates were estimated using the SPost suite of postestimation commands in Stata. All analyses were conducted using Stata 12.1 (StatCorp.) All P-values are two sided, and $P<.05$ was considered statistically significant.

\section{Results}

The flow of participants through our cohort study is shown in Fig. 1. A total of 504 women were recruited to the study and had serum collected for vitamin D assay. Four blood samples could not be processed for serum vitamin $\mathrm{D}$ status due to the blood sample being insufficient. Thirty-six recruited participants never commenced their planned assisted reproductive treatment. The remaining 464 participants commenced their treatment and had their reproductive treatment outcomes collected. Thirty-three participants had cycles abandoned at different stages of their IVF, ICSI and FET treatment either due to ovarian hyper-stimulation, poor ovarian response, failed fertilisation or no oocytes being collected at attempted retrieval. Four hundred and one study 


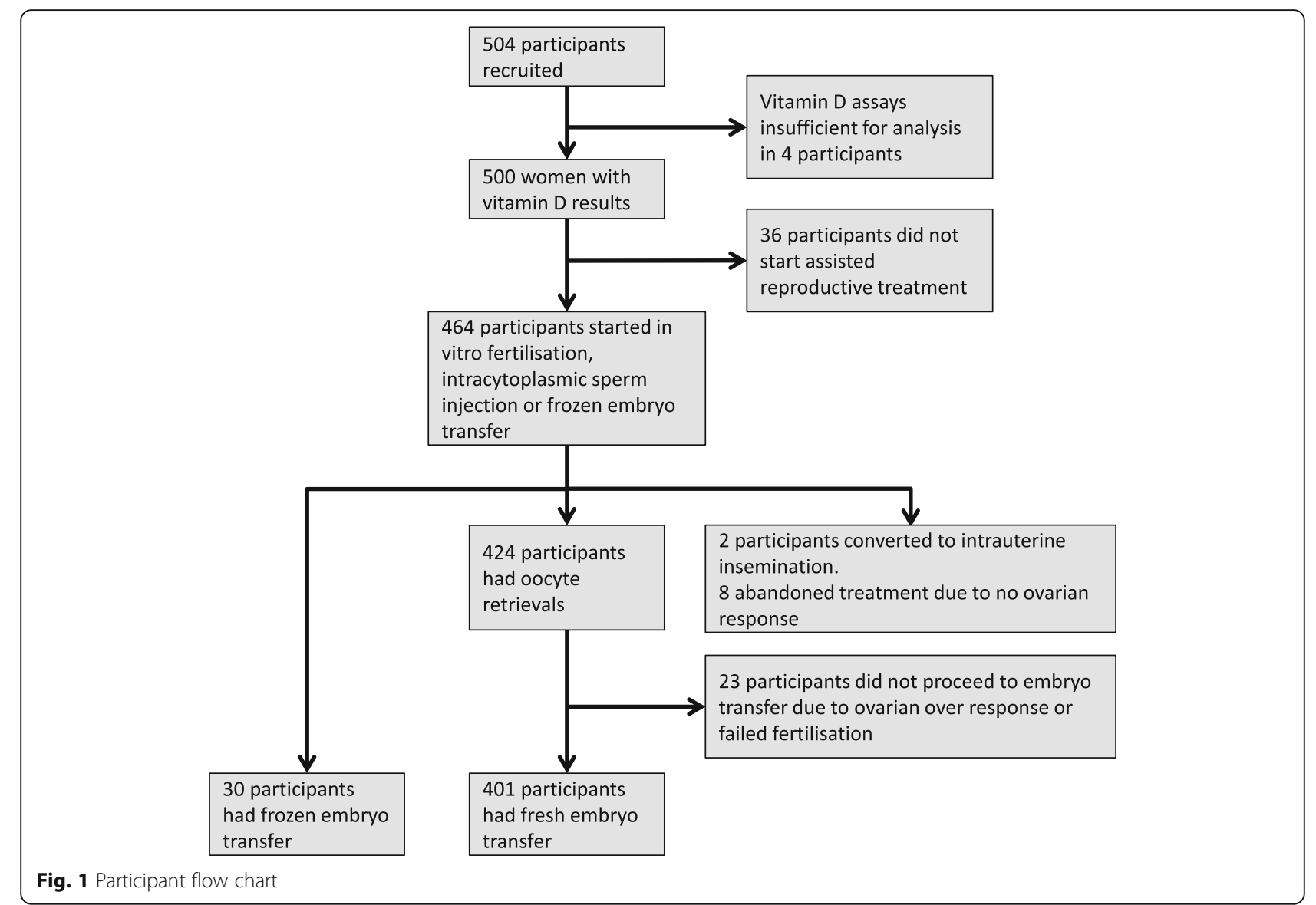

participants underwent fresh embryo transfer. Thirty participants underwent a frozen embryo transfer.

\section{Vitamin D status}

The vitamin D status of the 500 recruited participants is shown in Fig. 2. Vitamin D deficiency (total vitamin D 0 to $49.9 \mathrm{nmol} / \mathrm{L}$ ) affected 266 out of $500(53.2 \%)$ participants. Vitamin D insufficiency (total vitamin D 50 to $75 \mathrm{nmol} / \mathrm{L}$ ) affected 154 of 500 participants (30.8\%). Eighty out of the 500 participants $(16.0 \%)$ were replete in vitamin $\mathrm{D}(>75 \mathrm{nmol} / \mathrm{L})$.

\section{Baseline characteristics}

The baseline characteristics are shown in Table 1. The three vitamin D groups were similar in mean age $(p=0.20)$ and mean body mass index (BMI) for the three vitamin D groups was also comparable $(p=0.18)$. The serum vitamin D levels varied according to ethnicity. More South Asian and Black women were vitamin D deficient, 71.8 and $66.7 \%$ respectively. Vitamin D status was more evenly distributed amongst White participants (36.0\% deficient, 39.2\% insufficient and $24.8 \%$ replete). Vitamin D status was unevenly distributed when comparing differing ethnicities and this reached statistical significance $(p<0.001)$. A significant difference was found when comparing vitamin $\mathrm{D}$ status with the season. Vitamin D levels were more likely to be in the deficient range in the Winter (59.4\%) and Spring (61.6\%) when compared to Summer $(31.0 \%)(\mathrm{p}<0.001)$.

Smoking is an important prognostic factor in IVF treatment success. Only 26 of the participants smoked. The proportion of smokers in the vitamin D deficient, insufficient and replete groups were comparable $(p=$ 0.64).

The key fertility history variables are also displayed in Table 1 and additional information is provided in Additional file 1. Amongst the participants, the duration of infertility was found to be longer in the vitamin D deficient group when compared to the vitamin D insufficient and replete groups. Ovarian reserve, type of treatment, the proportion of women receiving a blastocyst, and the proportion of women undergoing single embryo transfer were similar in all three vitamin D categories.

\section{Pregnancy outcomes}

The ART outcomes are shown in Table 2 and Fig. 3. Live birth rates in the vitamin $\mathrm{D}$ groups were significantly different; the live birth rates in the deficient, insufficient and replete groups were $23.2 \%$ (95\% CI 18.0 to 28.4), $27.0 \%$ (95\% CI 19.6 to 34.3 ) and $37.7 \%$ (95\% CI 26.9 to $48.5)$ respectively $(p=0.04)$. 


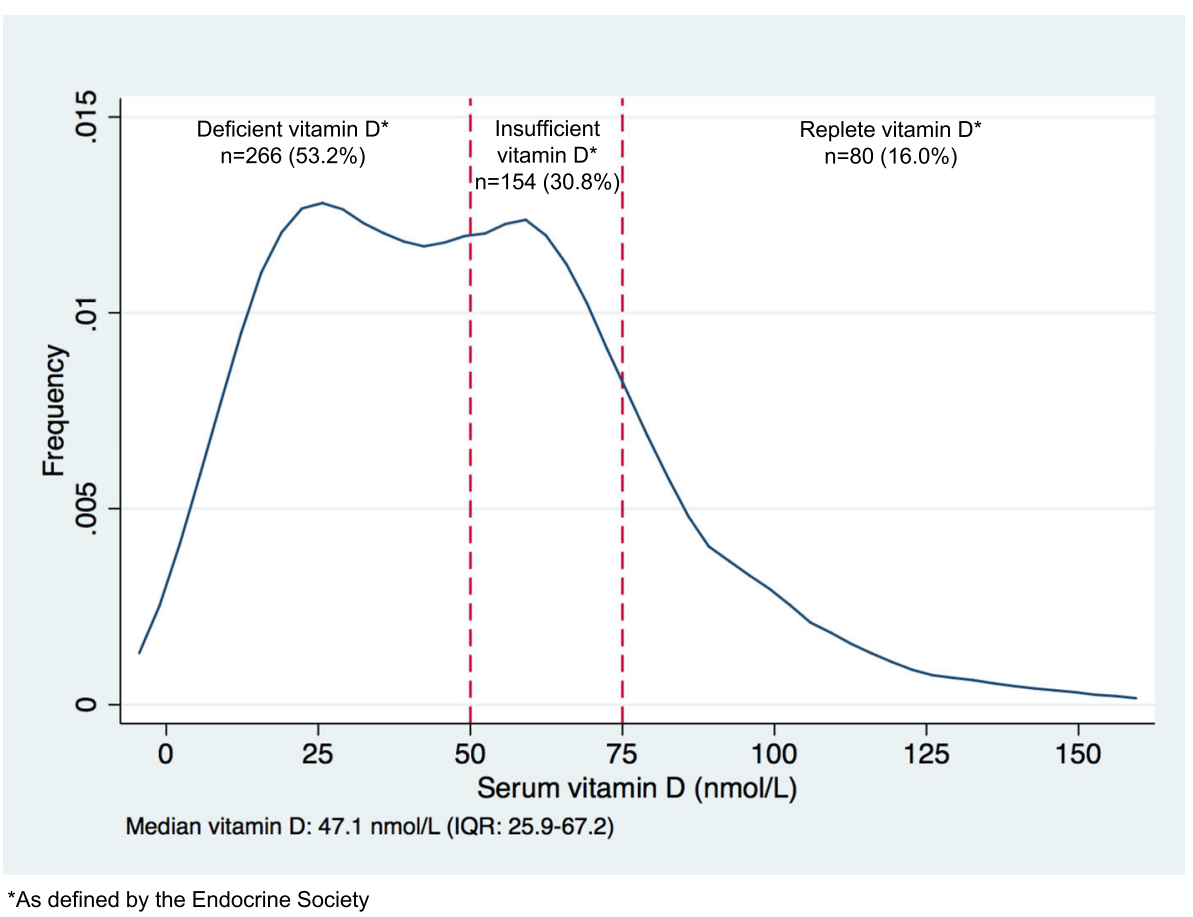

Fig. 2 Vitamin D levels for women undergoing assisted reproductive treatments

Biochemical pregnancy rates (the percentage of women in each vitamin D group achieving a positive pregnancy test two weeks after embryo transfer) in deficient, insufficient and replete groups were $32.5 \%$ (95\% CI 26.7 to 38.4 ), $39.0 \%$ (95\% CI 30.9 to 47.1 ) and $48.1 \%$ (95\% CI 36.9 to 59.2 ) respectively. The biochemical pregnancy rates show a statistically significant difference between the three groups $(p=$ $0.04)$. Of the women achieving a positive pregnancy test, the biochemical pregnancy loss rates (number of women miscarrying after having a positive pregnancy test and before having fetal heart activity seen on ultrasound scan) for vitamin $\mathrm{D}$ deficient, insufficient and replete vitamin groups were $20.0 \%$ ( $95 \%$ CI 11.2 to 28.8 ), $18.2 \%$ (95\% CI 8.0 to 28.4 ) and $13.5 \%$ (95\% CI 2.5 to 24.5$)$ respectively $(p=0.70)$.

The clinical pregnancy rates (the percentage of women achieving fetal heart activity five weeks after embryo transfer) in deficient, insufficient and replete groups were $26.0 \%$ (95\% CI 20.6 to 31.4 ), $31.9 \%$ (95\% CI 24.2 to 39.6), and $41.6 \%$ (95\% CI 30.5 to 52.6$)$ respectively. The trend in clinical pregnancy between the three groups is statistically significant $(p=0.03)$. Of the women achieving a clinical pregnancy, the clinical pregnancy miscarriage rates for vitamin $\mathrm{D}$ deficient, insufficient and replete groups were $10.9 \%$ (95\% CI 3.3 to 18.6 ), $15.6 \%$ (95\% CI 5.0 to 26.1 ), and $9.4 \%(95 \% \mathrm{CI} 0.1$ to 19.5$)$ respectively $(p=0.67)$.

\section{The white population}

A subgroup analysis of the IVF treatment outcomes in the White population was performed. This was carried out to ascertain whether vitamin D is an independent variable that affects IVF treatment outcome in our largest ethnic group. When comparing vitamin $\mathrm{D}$ deficient and insufficient white women with vitamin D replete white women, the live birth rates and clinical pregnancy rates showed clear trends; however, the trend did not reach statistical significance. The live birth rates in vitamin D deficient, insufficient and replete white women were $29.1,30.8$ and $43.6 \%$ respectively $(p=0.14)$. The clinical pregnancy rates in vitamin D deficient, insufficient and replete white women were 31.4, 35.2 and $46.8 \%$ respectively $(p=0.15)$.

\section{Multivariate analysis}

We performed a multivariate analysis to adjust for several confounding factors. Vitamin D is known to be affected by a patient's BMI and ethnicity [21]. Smoking, age, cause of infertility, duration of infertility, baseline pituitary FSH assay, type of assisted reproductive treatment performed, and ART protocol used are important prognostic factors associated with pregnancy outcome. The adjusted analysis of crude data from our cohort study shows that live birth, clinical pregnancy and biochemical pregnancy are more likely in women who are replete in vitamin $\mathrm{D}$ when compared to women with deficient or insufficient vitamin D levels although the difference in pregnancy outcome rates do not reach statistical significance (Table 2). 
Table 1 Baseline characteristics and treatment cycle variables of participants undergoing assisted reproductive treatments

\begin{tabular}{|c|c|c|c|c|}
\hline \multicolumn{4}{|l|}{ Vitamin D Category } & \multirow[t]{2}{*}{$p$-value } \\
\hline & $\begin{array}{l}\text { Deficient }^{a} \\
(<50 \mathrm{nmol} / \mathrm{L}) \\
N=266\end{array}$ & $\begin{array}{l}\text { Insufficient }^{\mathrm{a}} \\
(50-75 \mathrm{nmol} \mathrm{L}) \\
N=154\end{array}$ & $\begin{array}{l}\text { Replete }^{a} \\
(>75 \mathrm{nmol} / \mathrm{L}) \\
N=80\end{array}$ & \\
\hline Age -years(SD) & $33.2(4.9)$ & $33.7(4.4)$ & $34.2(4.6)$ & 0.20 \\
\hline BMI (SD) & $24.7(4.0)$ & $25.1(3.6)$ & $24.1(3.1)$ & 0.18 \\
\hline \multicolumn{5}{|l|}{ Ethnicity (\%) } \\
\hline White & $93(36.0)$ & $101(39.2)$ & $64(24.8)$ & \multirow[t]{5}{*}{$<0.001^{+}$} \\
\hline South Asian & $125(71.8)$ & 37 (21.3) & $12(6.9)$ & \\
\hline Black & $20(66.7)$ & $8(26.7)$ & $2(6.6)$ & \\
\hline Chinese & $5(62.5)$ & $2(25.0)$ & $1(12.5)$ & \\
\hline Other & $23(76.7)$ & $6(20.0)$ & $1(3.3)$ & \\
\hline \multicolumn{5}{|l|}{ Season (\%) } \\
\hline Winter & $76(59.4)$ & $41(32.0)$ & $11(8.6)$ & \multirow[t]{4}{*}{$<0.001^{+}$} \\
\hline Spring & 77 (61.6) & $39(31.2)$ & $9(7.2)$ & \\
\hline Summer & $31(31.0)$ & $40(40.0)$ & $29(29.0)$ & \\
\hline Autumn & $82(55.8)$ & $34(23.1)$ & $31(21.1)$ & \\
\hline \multicolumn{5}{|l|}{ Smoking (\%) } \\
\hline Smokers & $16(6.0)$ & $6(3.9)$ & $4(5.0)$ & $0.64^{+}$ \\
\hline Duration of infertility in months (Median-IQR) & $48(36-72)$ & $36(24-60)$ & $36(24-60)$ & 0.04 \\
\hline Mean pituitary FSH (iU/ml) (SD) & $7.9(2.9)$ & $7.6(2.9)$ & $7.9(4.0)$ & 0.73 \\
\hline Mean AMH (SD) & $18.7(21.7)$ & $22.6(27.4)$ & $14.3(14.1)$ & 0.34 \\
\hline Previous live birth (\%) & $198(80.5)$ & $11(78.7)$ & $62(80.5)$ & $0.91^{+}$ \\
\hline \multicolumn{5}{|l|}{ Treatment type (\%) } \\
\hline IVF & $99(40.2)$ & $54(38.3)$ & $40(52.0)$ & \multirow[t]{3}{*}{$0.37^{+}$} \\
\hline ICSI & $130(52.9)$ & $78(55.3)$ & $33(42.9)$ & \\
\hline FET & $17(6.9)$ & $9(6.4)$ & $4(5.1)$ & \\
\hline Number of embryos transferred at day 5 post oocyte retrieval (\%) & $97(46.6)$ & $65(53.3)$ & $40(56.3)$ & $0.28^{+}$ \\
\hline \multicolumn{5}{|l|}{ Number of embryos transferred (\%) } \\
\hline Single & $153(73.6)$ & $79(64.8)$ & $51(71.8)$ & \multirow[t]{2}{*}{$0.23^{+}$} \\
\hline Double & $55(26.4)$ & $43(35.2)$ & $20(28.2)$ & \\
\hline Top grade embryo transfer & $169(81.2)$ & $93(76.2)$ & $56(78.9)$ & $0.55^{+}$ \\
\hline
\end{tabular}

${ }^{\mathrm{a}}$ As defined by Endocrine Society

${ }^{+} p$-value for $\mathrm{chi}^{2}$ test

\section{Discussion}

\section{Main findings}

Our prospective cohort study indicates that vitamin D deficiency and insufficiency is highly prevalent among women undergoing assisted reproductive treatment. Furthermore, serum vitamin D status is associated with IVF outcomes. Our cohort study is the first to be performed in the UK and the findings confirm what other research groups [22-29] have found in other countries, that women who are vitamin $\mathrm{D}$ replete are more likely to achieve a live birth through their IVF treatment than those who are vitamin D deficient or insufficient. When adjusting for confounding factors in our multivariate analysis, trends in reproductive treatment outcomes

were maintained, however, statistical significance in live birth, clinical pregnancy and biochemical pregnancy rates was lost. When analysing ART outcomes in the white population (our largest ethnic group), a biological gradient was demonstrated with the highest live birth rates achieved in women replete in vitamin $\mathrm{D}$, and the lowest live birth rates achieved in women deficient in vitamin D.

\section{Strengths and limitations}

There were several strengths to our cohort study. Compared to other similar cohort studies, this study is large. This reduces the likelihood that the association that we found to be due to chance. Additionally, we had no 
Table 2 Outcomes for women undergoing assisted reproductive treatment

\begin{tabular}{|c|c|c|c|c|c|}
\hline & & $\begin{array}{l}\text { Deficient }^{\mathrm{a}} \\
<50 \mathrm{nmol} / \mathrm{L} \\
\mathrm{n} / \mathrm{N} \\
\%(95 \% \mathrm{Cl})\end{array}$ & $\begin{array}{l}\text { Insufficient }^{\mathrm{a}} \\
\left(50-75 \mathrm{nmol}^{\prime} \mathrm{L}\right) \\
\mathrm{n} / \mathrm{N} \\
\%(95 \% \mathrm{Cl})\end{array}$ & $\begin{array}{l}\text { Replete }^{a} \\
(>75 \mathrm{nmol} / \mathrm{L}) \\
\mathrm{n} / \mathrm{N} \\
\%(95 \% \mathrm{Cl})\end{array}$ & $p$-value \\
\hline \multirow[t]{2}{*}{ Live birth } & Crude rates & $\begin{array}{l}57 / 246 \\
23.2(18.0-28.4)\end{array}$ & $\begin{array}{l}38 / 141 \\
27.0(19.6-34.3)\end{array}$ & $\begin{array}{l}29 / 77 \\
37.7(26.9-48.5)\end{array}$ & 0.04 \\
\hline & Adjusted rates ${ }^{\mathrm{b}}$ & $24.3(18.7-29.9)$ & $27.1(19.9-34.2)$ & $34.4(23.9-44.9)$ & 0.25 \\
\hline \multirow[t]{2}{*}{$\begin{array}{l}\text { Positive pregnancy } \\
\text { test }\end{array}$} & Crude rates & $\begin{array}{l}80 / 246 \\
32.5(26.7-38.4)\end{array}$ & $\begin{array}{l}55 / 141 \\
39.0(30.9-47.1)\end{array}$ & $\begin{array}{l}37 / 77 \\
48.1(36.9-59.2)\end{array}$ & 0.04 \\
\hline & Adjusted rates ${ }^{\mathrm{b}}$ & $34.1(27.9-40.4)$ & $36.7(28.7-44.8)$ & $43.8(32.5-55.2)$ & 0.35 \\
\hline \multirow[t]{2}{*}{ Clinical pregnancy } & Crude rates & $\begin{array}{l}64 / 246 \\
26.0(20.6-31.4)\end{array}$ & $\begin{array}{l}45 / 141 \\
31.9(24.2-39.6)\end{array}$ & $\begin{array}{l}32 / 77 \\
41.6(30.5-52.6)\end{array}$ & 0.03 \\
\hline & Adjusted rates ${ }^{b}$ & $27.1(21.2-33.0)$ & $32.0(24.3-39.7)$ & $38.7(27.7-49.7)$ & 0.19 \\
\hline \multirow[t]{2}{*}{$\begin{array}{l}\text { Biochemical } \\
\text { pregnancy loss }\end{array}$} & Crude rates & $\begin{array}{l}16 / 80 \\
20.0(11.2-28.8)\end{array}$ & $\begin{array}{l}10 / 55 \\
18.2(8.0-28.4)\end{array}$ & $\begin{array}{l}5 / 37 \\
13.5(2.5-24.5)\end{array}$ & 0.70 \\
\hline & Adjusted rates ${ }^{b}$ & $21.6(11.6-31.5)$ & $16.1(5.7-26-5)$ & $14.2(2.2-26.1)$ & 0.64 \\
\hline \multirow[t]{2}{*}{$\begin{array}{l}\text { Clinical pregnancy } \\
\text { loss }\end{array}$} & Crude rates & $\begin{array}{l}7 / 64 \\
10.9(3.3-18.6)\end{array}$ & $\begin{array}{l}7 / 45 \\
15.6(5.0-26.1)\end{array}$ & $\begin{array}{l}3 / 32 \\
9.4(0.1-19.5)\end{array}$ & 0.67 \\
\hline & Adjusted rates ${ }^{\mathrm{b}}$ & $12.2(4.2-20.2)$ & $22.2(7.7-36.7)$ & $16.3(1.8-30.9)$ & 0.55 \\
\hline
\end{tabular}

${ }^{\mathrm{a}}$ As defined by Endocrine Society

${ }^{b}$ Adusted for age, body mass index, ethnicity, smoking status, cause of infertility, duration of infertility, baseline pituitary follicular stimulating hormone assay, treatment protocol, and type of treatment

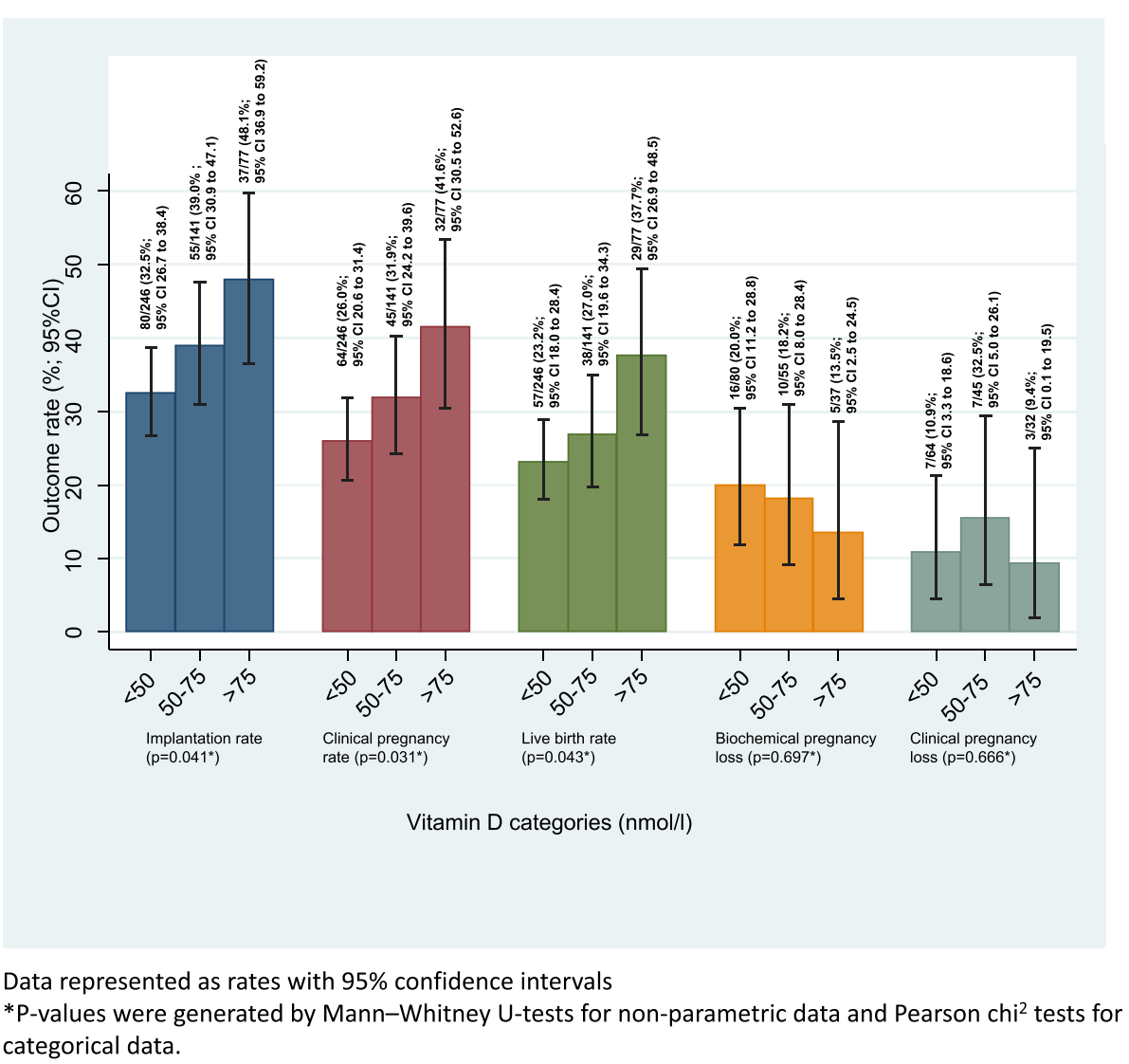

Fig. 3 Pregnancy outcomes for women undergoing assisted reproductive treatment by vitamin D levels. Data represented as rates with $95 \%$ confidence intervals 
exclusion criteria and aimed to include as many patients undergoing IVF, ICSI and FET treatments as possible. This enables our findings to have greater generalisability. Lastly, the population that we recruited was ethnically diverse, increasing representativeness for the rest of the UK population and specifically all other UK assisted reproductive treatment providers.

The cohort study also had weaknesses. The biological plausibility that vitamin D affects IVF treatment outcome appears to stem from its effects on the endometrium. Ideally, in order to prove the hypothesis that vitamin D exerts its effects on treatment outcome via the endometrium, serum for vitamin D assay should have been obtained on the day of embryo transfer. This ensures that vitamin D status is as close temporally to the time of embryo replacement as possible. In our study, women were approached at the start of their ART. Therefore, there may have been changes and fluctuations to the serum vitamin D level during ART after the assay was performed. However, fluctuations in vitamin D are only achieved when vitamin D deficiency or insufficiency is treated. Although many of the participants may have been taking preconception vitamins at the time the blood test was obtained, the supplementary doses are known to be not high enough to treat and correct vitamin D deficiency or insufficiency [17].

Another weakness in the cohort study is in its observational nature. Although an association between vitamin D and IVF treatment outcome is demonstrated, we are unable to conclude that vitamin D deficiency treatment improves outcomes; only an interventional trial would achieve this.

\section{Interpretation}

Statistical significance was lost when modifying for confounding factors in our multivariate analysis. The confounding factor that led to the loss of statistical significance in our multivariate model was ethnicity. This occurred due to the low numbers of women who were vitamin replete in the non-white ethnic groups. Importantly, despite the loss of statistical significance in our adjusted analysis, the trend of lowest biochemical, clinical and live birth rates in the vitamin D deficient group and the highest biochemical, clinical and live birth rates were maintained.

Interestingly, in our cohort study, when the ART outcomes were analysed in isolation in the white population, women with replete vitamin D levels achieved an increased rate of clinical pregnancy and live birth when compared to those with vitamin D deficiency/insufficiency. Although strong conclusions cannot be drawn from observational data, this would suggest that in white women, vitamin D could play an important independent role in predicting IVF treatment success. This could be via its actions within the endometrium promoting pregnancy implantation or as a surrogate marker for lifestyle and general health.

In our cohort study, the data shows that if participants are able to achieve implantation (by achieving a positive pregnancy test) the chances of the implanted embryo progressing to become a clinical pregnancy and a live birth are comparable between the three groups (Fig. 4). This supports the theory that vitamin D's function in fertility is mainly in initial implantation of the embryo.

Seasonal variations in natural conception rates have already been established [30] with higher conception rates found in the Summer and Autumn. Although many hypotheses have been postulated to explain this phenomenon (e.g. reduced ovulation rates and poorer sperm quality in darker months) the exact mechanism behind this has not been explained. It is possible that an increase in sun exposure and greater sunlight luminosity increases the body's store of vitamin D, thereby yielding higher natural conception rates in Summer and Autumn.

Although the debate regarding the importance of vitamin D and seasonal variation in reproductive health continues, its impact on immunomodulation within the endometrium with a resultant reduction of active inflammatory cytokines is now well understood [16]. The expression of vitamin $\mathrm{D}$ receptors at the level of the endometrium and the role of vitamin D in the transcription of HOX10A gene (found to be of key importance in implantation) suggest that the immunomodulatory effects of vitamin D may have a direct impact on implantation and therefore the likelihood of reproductive treatment success.

Other studies have investigated the link between vitamin D status and implantation further. Some research groups have attempted to isolate the effects of vitamin D on the endometrium and implantation by studying women undergoing donor oocyte IVF treatment cycles. In such cycles, one would expect all oocyte prognostic determinants to be nullified, as only high quality oocytes are used. In effect endometrial receptivity and implantation alone are tested. Examples of such studies include the study by Fabris et al., [31] (who did not find an association between vitamin $\mathrm{D}$ and implantation in women undergoing IVF treatment using donor oocytes), and the study by Rudick et al., [22] who found a strong association between the vitamin $\mathrm{D}$ status of donor oocyte recipients and the IVF treatment success.

In other published research, ethnicity has been found to be a prognostic marker for IVF treatment success on its own [32]. Perhaps the reason for this could be due to higher prevalence of vitamin D deficiency in these ethnic groups due to darker pigmented skin, absorbing a lower level of ultraviolet light in the UK. Consequently, this 


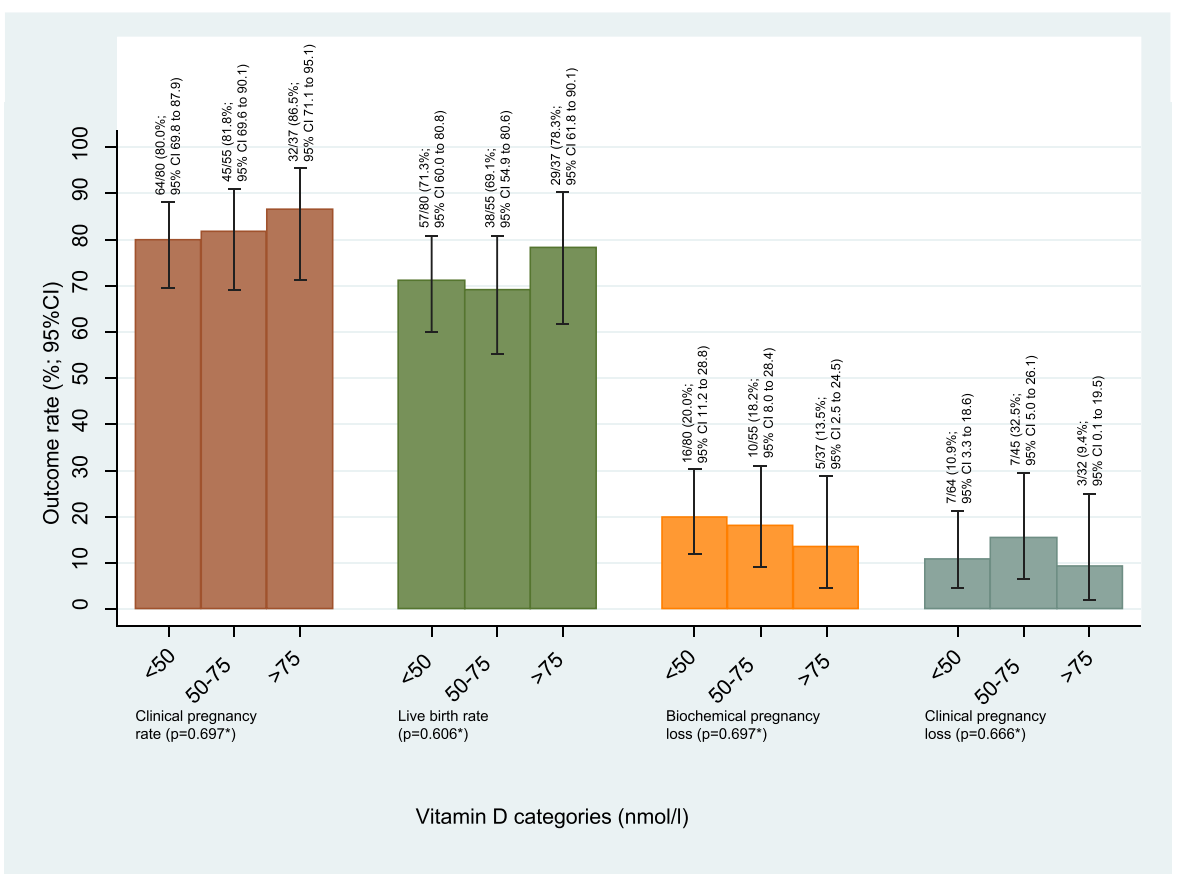

Data represented as rates with $95 \%$ confidence intervals

*P-values were generated by Mann-Whitney U-tests for non-parametric data and Pearson chi² tests for categorical data.

Fig. 4 Outcomes for women who achieved a positive pregnancy test with assisted reproductive treatment

would reduce the stores of vitamin D in Asian and Black women. Vitamin D receptor gene polymorphisms have already been identified in the Asian population, which may act as a confounding or modifying factor [33, 34].

\section{Conclusion}

In summary, vitamin $\mathrm{D}$ deficiency may be a treatable factor that can potentially improve the chances of ART success. Vitamin D serum testing is relatively cheap and widely available. Furthermore, treatment of deficiency or insufficiency with subsequent maintenance therapy is not costly and could also reduce the risk of obstetric complications such as gestational diabetes, preeclampsia, and fetal growth restriction. However, an interventional trial may be necessary to establish the effects of vitamin D treatment on assisted reproductive treatment outcomes.

\section{Additional file}

Additional file 1: Additional assisted reproductive treatment cycle variables of cohort study participants. *As defined by Endocrine Society. ${ }^{+} p$-value for chi2 test. (DOCX $59 \mathrm{~kb}$ )

\section{Abbreviations}

ART: Assisted reproductive treatments; BMI: Body mass index; FET: Frozen embryo transfer; FSH: Follicular stimulating hormone; ICSI: Intra-cytoplasmic sperm injection; IVF: In vitro fertilisation; NRES: National research ethics service; UK: United Kingdom

\section{Acknowledgments}

Not applicable

\section{Authors' contributions}

$J C$ led the funding application, with input from $A C, L R$ and JKB. JC, RD, RR, $\mathrm{AE}$ and $\mathrm{HH}$ performed participant recruitment and data collection. Statistical analyses were performed by AT and IDG. JC wrote the first draft of the manuscript and is its guarantor. All authors revised it critically for important intellectual content, gave final approval of the version to be published.

\section{Funding}

The cohort study was funded by a grant from the Research and Development Department at the Birmingham Women's and Children's NHS Foundation Trust.

\section{Availability of data and materials}

All data generated or analysed during this study are included in this published article [and its additional file].

\section{Ethics approval and consent to participate}

This study was approved by the National Research Ethics Service (NRES) Committee West Midlands - Black Country (REC 13/WM/0258). All participants provided full written and informed consent.

\section{Consent for publication}

Not applicable

\section{Competing interests}

The authors declare that they have no competing interests. 


\section{Author details}

${ }^{1} T o m m y$ 's National Centre for Miscarriage Research, Institute of Metabolism and Systems Research (IMSR), University of Birmingham, Birmingham B15 2TT, UK. 'Birmingham Women's and Children's National Foundation Trust, Mindelsohn Way, Birmingham B15 2TG, UK. ${ }^{3}$ Spanish Council for Scientific Research, Institute of Environmental Assessment and Water Research, Barcelona, Spain. ${ }^{4}$ Birmingham Women's Foundation NHS Trust, Edgbaston B15 2TG, UK

Received: 24 September 2018 Accepted: 30 June 2019

Published online: 15 July 2019

\section{References}

1. HFEA. Fertility treatment in 2013. Trends and figures. 2013.

2. Grady R, Alavi N, Vale R, Khandwala M, McDonald SD. Elective single embryo transfer and perinatal outcomes: a systematic review and metaanalysis. Fertil Steril. 2012:324-31.

3. Macklon NS, Geraedts JPM, Fauser BCJM. Conception to ongoing pregnancy: the "black box" of early pregnancy loss. Hum Reprod Update. 2002:8:333-43.

4. Gordon CM, DePeter KC, H a F, Grace E, Emans SJ. Prevalence of vitamin D deficiency among healthy adolescents. Arch Pediatr Adolesc Med. 2004;158: 531-7.

5. Sullivan SS, Rosen CJ, Halteman WA, Chen TC, Holick MF. Adolescent girls in Maine are at risk for vitamin D insufficiency. J Am Diet Assoc. 2005;105: 971-4.

6. Tangpricha V, Pearce EN, Chen TC, Holick MF. Vitamin D insufficiency among free living healthy young adults. Am J Med. 2002;112:659-62.

7. Lerchbaum E, Rabe T. Vitamin D and female fertility. Curr Opin Obstet Gynecol [Internet]. 2014;26:145-50 Available from: http://www.ncbi.nlm.nih. gov/pubmed/24717915

8. Halloran BP, DeLuca HF. Effect of vitamin D deficiency on fertility and reproductive capacity in the female rat. J Nutr. 1980;110:1573-80.

9. Kinuta K, Tanaka H, Moriwake T, Aya K, Kato S, Seino Y. Vitamin D is an important factor in estrogen biosynthesis of both female and male gonads. Endocrinology. 2000;141:1317-24.

10. Yoshizawa T, Handa Y, Uematsu Y, Takeda S, Sekine $K$, Yoshihara Y, et al. Mice lacking the vitamin $D$ receptor exhibit impaired bone formation, uterine hypoplasia and growth retardation after weaning. Nat Genet. 1997; 16:391-6. Available from: https://www.ncbi.nlm.nih.gov/pubmed/9241280, http://www.nature.com/ng/journal/v16/n4/abs/ng0897-391.html.

11. Panda DK, Miao D, Tremblay ML, Sirois J, Farookhi R, Hendy GN, et al. Targeted ablation of the 25-hydroxyvitamin D 1alpha -hydroxylase enzyme: evidence for skeletal, reproductive, and immune dysfunction. Proc Natl Acad Sci U S A. 2001;98:7498-503.

12. Aghajafari F, Nagulesapillai T, Ronksley PE, Tough SC, O'Beirne M, Rabi DM. Association between maternal serum 25-hydroxyvitamin D level and pregnancy and neonatal outcomes: systematic review and meta-analysis of observational studies. BMJ [Internet]. 2013;346:f1169 Available from: https:// www.ncbi.nlm.nih.gov/pubmed/23533188, http://www.bmj.com/content/ bmj/346/bmj.f1169.full.pdf.

13. Bodnar LM, Catov JM, Simhan HN, Holick MF, Powers RW, Roberts JM Maternal vitamin D deficiency increases the risk of preeclampsia. J Clin Endocrinol Metab. 2007;92:3517-22. Available from: https://www.ncbi.nlm. nih.gov/pubmed/17535985.

14. Baker AM, Haeri S, Camargo CA, Espinola JA, Stuebe AM. A nested casecontrol study of Midgestation vitamin D deficiency and risk of severe preeclampsia. J Clin Endocrinol Metab. 2010;95:5105-9 Available from: https://www.ncbi.nlm.nih.gov/pubmed/20719829.

15. Robinson CJ, Wagner CL, Hollis BW, Baatz JE, Johnson DD. Maternal vitamin $\mathrm{D}$ and fetal growth in early-onset severe preeclampsia. Am J Obstet Gynecol. 2011;204.

16. Holick MF. Vitamin D deficiency. N Engl J Med [Internet]. 2007;357:266-81 Available from: http://www.ncbi.nlm.nih.gov/pubmed/17634462.

17. Holick MF, Binkley NC, H a B-F, Gordon CM, D a H, Heaney RP, et al. Evaluation, treatment, and prevention of vitamin D deficiency: an Endocrine Society clinical practice guideline. J Clin Endocrinol Metab. 2011;96:1911-30 Available from: http://www.ncbi.nlm.nih.gov/pubmed/21646368.

18. Heaney RP. Vitamin D in health and disease. Clin J Am Soc Nephrol [Internet]. 2008:3:1535-41 Available from: http://www.ncbi.nlm.nih.gov/ pubmed/18525006.
19. Stephanou A, Ross R, Handwerger S. Regulation of human placental Lactogen expression by 1,25-Dihydroxyvitamin D3. Endocrinology. 1994;135: 2651-6.

20. Daftary GS, Taylor HS. Endocrine regulation of HOX genes. Endocr Rev. 2006: 331-55.

21. Holick MF, Chen TC. Vitamin D deficiency: a worldwide problem with health consequences. Am J Clin Nutr. 2008;87.

22. Rudick BJ, Ingles SA, Chung K, Stanczyk FZ, Paulson RJ, Bendikson KA. Influence of vitamin $D$ levels on in vitro fertilization outcomes in donorrecipient cycles. Fertil Steril. 2014;101:447-52.

23. Rudick B, Ingles S, Chung K, Stanczyk F, Paulson R, Bendikson K Characterizing the influence of vitamin $D$ levels on IVF outcomes. Hum Reprod. 2012;27:3321-7.

24. Ozkan S, Jindal S, Greenseid K, Shu J, Zeitlian G, Hickmon C, et al. Replete vitamin D stores predict reproductive success following in vitro fertilization. Fertil Steril. 2010;94:1314-9.

25. Garbedian K, Boggild M, Moody J, Liu KE. Effect of vitamin D status on clinical pregnancy rates following in vitro fertilization. C Open. 2013;1:E7782 Available from: http://www.ncbi.nlm.nih.gov/pubmed/25077107.

26. Paffoni A, Ferrari S, Viganò P, Pagliardini L, Papaleo E, Candiani M, et al. Vitamin D deficiency and infertility: insights from in vitro fertilization cycles. I Clin Endocrinol Metab. 2014;99:E2372-6.

27. Farzadi L, Khayatzadeh Bidgoli H, Ghojazadeh M, Bahrami Z, Fattahi A, Latifi Z, Shahnazi VNM. Correlation between follicular fluid 25-OH vitamin D and assisted reproductive outcomes. Iran J Reprod Med. 2015;13:361-6.

28. Polyzos NP, Anckaert E, Guzman L, Schiettecatte J, Van Landuyt L, Camus M, et al. Vitamin D deficiency and pregnancy rates in women undergoing single embryo, blastocyst stage, transfer (SET) for IVF/ICSI. Hum Reprod. 2014;29:2032-40.

29. Potashnik G, Lunenfeld E, Levitas E, Itskovitz J, Albutiano S, Yankowitz N, et al. The relationship between endogenous oestradiol and vitamin D3 metabolites in serum and follicular fluid during ovarian stimulation for invitro fertilization and embryo transfer. Hum Reprod. 1992:7:1357-60.

30. Rojansky N, Brzezinski A, Schenker JG. Seasonality in human reproduction: an update. Hum Reprod. 1992;7:735-45.

31. Fabris A, Pacheco A, Cruz M, Puente JM, Fatemi H, Garcia-Velasco JA. Impact of circulating levels of total and bioavailable serum vitamin D on pregnancy rate in egg donation recipients. Fertil Steril. 2014;102:1608-12.

32. Dhillon RK, McLernon DJ, Smith PP, Fishel S, Dowell K, Deeks JJ, et al. Predicting the chance of live birth for women undergoing IVF: a novel pretreatment counselling tool. Hum Reprod. 2016;31:84-92 Available from: http://humrep.oxfordjournals.org/lookup/doi/10.1093/humrep/dev268.

33. Ingles SA. Can diet and/or sunlight modify the relationship between vitamin D receptor polymorphisms and prostate Cancer risk? Nutr Rev. 2007;65.

34. John EM, Schwartz GG, Koo J, Wang W, Ingles SA. Sun exposure, vitamin D receptor gene polymorphisms, and breast cancer risk in a multiethnic population. Am J Epidemiol. 2007:166:1409-19.

\section{Publisher's Note}

Springer Nature remains neutral with regard to jurisdictional claims in published maps and institutional affiliations.

Ready to submit your research? Choose BMC and benefit from:

- fast, convenient online submission

- thorough peer review by experienced researchers in your field

- rapid publication on acceptance

- support for research data, including large and complex data types

- gold Open Access which fosters wider collaboration and increased citations

- maximum visibility for your research: over $100 \mathrm{M}$ website views per year

At BMC, research is always in progress.

Learn more biomedcentral.com/submission 